SSC00-II-1

\title{
NESS: Using a Microsatellite to Search For and Track Satellites and Asteroids
}

\author{
Dr. Kieran A. Carroll \\ Manager, Space Projects, Dynacon Enterprises Limited \\ 3565 Nashua Drive, Mississauga, Ontario, Canada L4V 1R1 \\ 905-672-8828 x232, kac@ dynacon.ca
}

Dr. Alan R. Hildebrand

Associate Professor, Department of Geology and Geophysics, University of Calgary

Room 760A, Earth Sciences Building, 2500 University Drive NW, Calgary, Alberta, Canada

T2N $1 \mathrm{~N} 4$

403-220-2291, hildebra@ geo.ucalgary.ca

David Balam

Department of Physics and Astronomy, University of Victoria

P. O. Box 3055, Victoria, British Columbia, Canada V8W 3P6

250-721-7749, cosmos@ uvvm.uvic.ca

Dr. Jaymie Matthews

Assistant Professor, Department of Physics and Astronomy, University of British Columbia 129 - 2219 Main Mall, Vancouver, British Columbia, Canada V6T 1Z4

604-822-2696, matthews@astro.ubc.ca

\begin{abstract}
The Near Earth Space Surveillance (NESS) mission is being developed by Dynacon and a team of asteroid scientists, supported by the Canadian Space Agency (CSA) and the Canadian Department of National Defence (DND). NESS uses a single satellite to perform a dual mission: searching for an tracking Earth-approaching asteroids, and tracking satellites in Earth orbit. There are aspects of both of these activities that are best accomplished using an orbiting observatory. The concept presented here is to implement NESS using a small imaging telescope mounted on a lowcost microsatellite-class platform, based on the design developed for the MOST stellar photometry microsatellite mission.
\end{abstract}

\section{Introduction}

The capabilities of microsatellites continue to grow more sophisticated with each passing year, allowing them to be used to carry out types of missions that once required much larger satellite platforms. In the early 1990s, miniaturization of digital electronics enabled microsats to demonstrate orbiting store-and-forward packet radio communications. Miniaturization of optical sensors then allowed optical remote sensing to be carried out from this class of platform.
Up until recently, imaging space science missions still relied on larger, more expensive satellites. However, the development of miniaturized attitude control system components such as reaction wheels and star trackers, has allowed a team led by Dynacon to develop the MOST mission ${ }^{1,2}$ for the CSA, in which a microsatellite-based space telescope employs an attitude control system ${ }^{3}$ similar to that of the Hubble space telescope.

These new capabilities also enable other types of missions to take advantage of the benefits of 
using the microsatellite approach-such as shorter development times, and much lower costs than for larger satellites - while achieving high levels of performance and capability. This paper summarizes one such, the Near Earth Space Surveillance mission.

We begin by explaining the rationale for the two objectives of the NESS mission, and for achieving them with a single satellite. The driving requirements for the mission are set out. (This paper will focus mainly on the rationale and requirements for the asteroid-science element of NESS; the information provided on the satellite-tracking is at more of an overview level.) A system design to achieve those requirements, based on the MOST design, is summarized. Key elements of the plan for developing this system are outlined.

\section{Mission Rationale}

The rationale for the asteroid-science aspect of the NESS mission is described in detail in this section. This is followed by a summary of the rationale for the satellite-tracking aspect of the mission.

\section{Mission \#1: Asteroid Science}

NESS proposes to use a space-based telescope to observe asteroids, in order to extract scientific knowledge from those observations. This is, of course, an activity that can be (and is) carried out using ground-based telescopes. Being located on the ground, however, imposes some limitations that interfere with the ability of scientists to make some very important types of observations; by being in orbit, NESS is able to fill these observational gaps. The overall context of existing ground-based asteroid observational astronomy is described below, as a prelude to describing the potential science contributions that can be made by NESS.

\section{Science Context}

Asteroids, the smallest planetary objects in the Solar System, are studied for several important reasons. Until recently, they could only be studied from afar by astronomers, who have discovered them one by one, and measured their orbits, their brightness and their reflectance spectra.

With the advent of planetary exploration space missions, such as NASA's current Near Earth Asteroid Rendezvous (NEAR) mission (http://near.jhuapl.edu/), spacecraft are underway to inspect some asteroids directly, collect from them and analyze samples, and eventually return samples to Earth for detailed study. This science has already resulted in an improved understanding of the evolution of the solar system, and of the genesis of Earth and the other major planets, and much more remains to be discovered; for example, asteroid and comet collisions may have played an important role in the genesis of life on Earth (http://neo.jpl.nasa.gov/neo.html).

In addition to their scientific value, some asteroids represent a threat to the Earth; there are many Near Earth Asteroids (NEAs) which follow orbits that bring them close to the Earth, and there is ample evidence that asteroids of all sizes have collided with the Earth throughout history, causing damage and climatic disruptions ranging up to the level of worldwide mass extinctions (e.g., see ht tp: //wwwdsa.uqac.uquebec.ca/ $\sim$ mhiggins/MIAC/chicxulub.htm). Recent international efforts have greatly improved the ability to predict far in advance how closely large asteroids will approach the Earth, potentially providing enough advance warning to allow action to be taken to avoid damage from such collisions, making this area of science potentially of the greatest possible practical importance to the entire population of the Earth.

The international NEA effort is carried out by a 
community of researchers who perform three broad tasks. The first is performing search programs in order to discover new NEAs. The second is carrying out astrometric and photometric data processing on these discovery observations, followed by computation of orbital elements and rapid dissemination of these elements to many follow-up observers. This enables the third task, rapid astrometric confirmation and follow-up of the discoveries, in the absence of which newly-discovered NEAs would promptly be "lost" again. In addition to these NEA-focused tasks, some astronomers also perform photometric, spectrographic and polarimetric observations of asteroids of all types, from which information on asteroid size, albedo, spin rate and composition can be deduced.

There are now several major NEA discovery programs in operation, using CCD detectors on large-aperture telescopes, principally the Spacewatch program (University of Arizona), NEAT (NASA-JPL), LINEAR (MIT Lincoln Laboratory), LONEOS (Lowell Observatory), the Catalina Sky Survey (University of Arizona) and BAO-SCAP (Beijing Astronomical Observatory). NEAT, LINEAR, LONEOS and Catalina are robotic telescopes that generate massive amounts of astrometric data; they are currently discovering 15-20 new NEAs per month. As a side-benefit, they are also discovering enormous numbers (tens of thousands per month!) of "main-belt" asteroids and comets. Processing of the massive amounts of astrometric and photometric data and calculation of orbital elements and ephemerides, as well as the dissemination of that data to the observational teams, is handled in an efficient and timely fashion by the Minor Planet Center of the IAU. The Spaceguard Central Node (Italy) and NASA-JPL use this orbital data to determine future (and past) close planetary encounters of all PHA's. Follow-up is done by a relatively small number of professional astronomers, and a much larger number of skilled amateurs.
NEAs come in different types, and NESS is most useful in making observations of some (not all) of these. A quick review of NEA nomenclature will help in the subsequent description of this: NEAs are classified by their orbit parameters, each named after the first asteroid discovered in each class. From http://neo.jpl.nasa.gov/neo.html, "Atens" (named after asteroid 2062 Aten) are Earthcrossing NEAs with semi-major axes smaller than Earth's and aphelions larger than 0.983 Astronomical Units (A.U.); 65 have been discovered. "Apollos" (named after asteroid 1862 Apollo) are Earth-crossing NEAs with semi-major axes larger than Earth's and perihelions smaller than 1.017 A.U.; 473 are currently known. There are currently 378 known "Amors" (named after asteroid 1221 Amor), Earth-approaching NEAs with orbits exterior to Earth's but interior to Mars'. "Potentially Hazardous Asteriods" (PHAs) are NEAs whose Minimum Orbit Intersection Distance (MOID) with the Earth is $0.05 \mathrm{AU}$ or less; 223 of magnitude brighter than 22 are known, 68 of which are brighter than magnitude 18. A total of 918 NEAs are currently known, 358 being brighter than magnitude 18 .

\section{Potential NESS Science Opportunities}

Aten-class NEA Search: A space-based observatory has a very great advantage over observatories on the ground, when used to search for Atens. This is because most of the time Atens are closer to the Sun than the Earth is, and so a telescope must point less than 90 degrees away from the Sun's direction to track them (that is, the target's "phase angle" is $>90$ degrees). When this is done from the ground, proximity of the Sun to the telescope's line of sight causes sky-glow, which obscures all but the brightest, closest objects. This may explain, at least partly, why the known population of Atens comprises a mere $7 \%$ of the known population of the near-Earth asteroids: there may be nearly as many Atens as Apollos, but because Atens are hard to observe with current 
ground-based instruments, most of them may go undetected. However, a telescope in Earth orbit does not have to contend with sky glow; while scattered light is an issue, suitable baffle design can allow such an instrument to point quite close to the Sun's direction (perhaps closer than 45 degrees), while maintaining a very dark image background level.

The observed population of NEAs is highly biased toward the most easily observed objects. The current sample of NEAs is complete only for objects in the $8 \mathrm{~km}$ diameter range. The level of completeness for asteroids in the $1 \mathrm{~km}$ range is estimated as $12 \%$. Observational methods are unreliable in determining an accurate estimate of the number of objects less than $1 \mathrm{~km}$ in diameter (sub-critical impactors).

NEA populations may be estimated by extrapolation from the known sample of objects and combined with calculated dynamical lifetimes to produce estimates of the numbers of objects in each size range; de-biasing these results in a population estimate of $2.6 \times 10^{5}$ NEAs in the range of diameter 100-1000 metres. The total number of critical impactors (diameter $>1 \mathrm{~km}$ ) is expected to be 1000-3000. If the Aten population accounts for about $25 \%$ of the NEA sample then we would expect there to be several hundred Atens larger than $1 \mathrm{~km}$ in diameter and perhaps 60,000 in the $100-1000 \mathrm{~m}$ range, providing a strong likelihood of NESS making numerous discoveries of new Aten-class NEAs. In addition, theoretical investigations of the long-term orbital behavior of NEAs have shown that Aten objects may dynamically evolve into orbits that are completely interior of the Earth's orbit (as "Inner Earth Objects" (IEOs), or Arjunas); NESS could have the capability of detecting these hypothetical objects.

NEA Rapid Astrometric Confirmation and Follow-up: While many newly-discovered NEAs can be followed-up using ground-based instruments, these are relatively poor at doing follow-up tracking of NEAs with aphelions close to the Sun that of Earth's orbit. This is due to the short time spent by such objects at opposition to the Earth before disappearing into the dawn sky; objects that are discovered during extreme close encounter (by definition, these are Potentially Hazardous Asteroids) will traverse the night sky in a matter of days (or in some cases hours!) and fade into the daylight sky before a fully-reliable orbital solution can be attained.

An orbiting telescope such as NESS would be able to track this type of object effectively for a considerably longer time. Note that objects of this type tend to suffer large numbers of close Earth encounters before actually impacting the planet, and predicting their orbit's evolution with time requires numerous observations over a long period of time; only a space-based astrometric telescope could provide the observations necessary to keep track of these objects.

Ground-based instruments are also completely incapable of tracking asteroids whose aphelions are less than that of Earth's orbit. These classes of objects represent an important segment of the NEA population for which only NESS would be able to perform astrometric observations.

Compositional studies: Analysis of visual and near-infrared spectral reflectances and spectral albedos provide the primary means of characterizing the surface mineralogy and petrology of asteroidal bodies. Ground-based colorimetry has contributed much toward understanding the mineralogy of the NEAs; to date, however, only a dozen Aten asteroids have been studied in this way. Addition of an ECAS (Eight Colour Asteroid Survey) broad-band filter system would allow NESS to be used to determine the mineralogical properties of an asteroid simultaneously with the acquisition of astrometric data. The further addition of photometric/polarimetric sensing capabilities could allow NESS to determine asteroid spin 
rates, sizes, albedos and compositions, for NEAs that are inaccessible to existing groundbased observatories.

\section{Mission \#2: Satellite Tracking}

Canada and the United States are partners in NORAD, the North American Aerospace Defense Command. NORAD historically has used the satellite-tracking services that are now provided by the U.S. Space Command, to distinguish between ballistic missiles approaching North America, and the $>23,000$ detectable man-made objects currently orbiting the Earth. This satellite tracking function is fed by data collected by the radar and optical sensors of the Space Surveillance Network (SSN), which continually measure the distance and/or direction from each sensor to satellites passing overhead.

In the past, Canada contributed sensors to this data-collection activity-a set of Baker-Nunn optical film cameras located in Canada. With the advent of electronic imaging sensors, the Baker-Nunn instruments have been decommissioned, replaced by new instruments such as GEODSS. Canada's Department of National Defence has recently initiated a Surveillance of Space (SOS) program, in order to contribute new Canadian sensors to the SSN.

Ground-based optical sensors are essentially optical telescopes, which take images of patches of the sky, which are analyzed to find the moving star-like images of satellites. There are several limitations that constrain the operations of any such sensor:

- They cannot operate during day-time, or too close to sunrise or sunset, due to sky-glow caused by the Sun obscuring the faint images of the satellites being tracked.

- Their effectiveness can be diminished at times when the Moon is in the sky, again due to sky-glow.
- Their effectiveness is diminished by cloudy or misty weather, frequently to the point of zero effectiveness.

- Less than half of the sky is visible to the sensor at any one time, due to obscuration by the Earth.

For these reasons, the amount of useful operational time from any one optical sensor can be very low, depending somewhat on geographic placement. Another significant geographic factor is that sensors located outside of Canada's borders would be difficult to maintain and operate, for logistical reasons.

It is notable that several of these limitations are not applicable to a space-based optical sensor, and the constraints imposed by the remaining limitations are significantly reduced. An orbiting optical sensor could be much more productive than a ground-based one, as well has having a much more reliable and rapid response time.

One question that arises naturally relates to telescope aperture size: can a telescope with sufficient photometric sensitivity to carry out a useful satellite-tracking mission, be built and flown within the small size constraints of an affordably-small satellite? The answer is "yes," as has been demonstrated by the Space Based Visible (SBV) experimental telescope ${ }^{4}$, flown as a payload aboard the BMDO's Midcourse Space Experiment (MSX) satellite in 1996. This telescope has a $15-\mathrm{cm}$ aperture-interestingly, the same aperture size as for MOST's telescope-and is routinely detecting satellites as faint as $M=15$. The sensor's performance is good enough that, after its experimental phase concluded successfully, the Space Surveillance Network began using it as a Contributing Sensor.

For these reasons, in addition to including several new ground-based optical sensors, the DND's SOS program will also involve Canada launching a satellite system to be used to track 
other satellites. This element of the Canadian SOS system will likely primarily do routine tracking of communications satellites in geostationary orbit, and other high-orbit Earth satellites (which in NORAD parlance are called "Deep Space Objects").

The schedule for this program involves placing one or mor tracking satellites into orbit around 2005. DND research is currently being carried out in support of this program, in order to improve the program's understanding of the mission requirements and design issues.

Based on the MOST satellite's precise photometry and imaging capabilities, the DND has developed an interest in the use of microsatellites to conduct some of this research in a rapid, low-cost way. The feasibility of doing this has been reinforced by the striking similarities between the MOST and SBV instruments, and the similarity in attitude control performance between MOST and the MSX satellite.

This has led to the concept of DND participating in the NESS mission, to carry out research in support of the development of SOS system requirements. (To pick just one of many examples, fundamental design decisions such as the question of how much image processing for target detection should be done on the ground versus on the satellite, depend critically on difficult-to-predict details of pixel-level imager noise characteristics. Flying an engineering model imager in the relevant environment, i.e. in orbit, would be a powerful way to validate analytical models of this type of effect.) This concept places a schedule requirement on the NESS mission: that it be developed promptly enough so that flight test results are available in time to be used by the designers of the operational SOS system.

\section{Mission Requirements}

Both the asteroid science and satellite tracking missions place their own requirements on the NESS system. The science requirements are discussed in detail in this section, and a summary is provided of the satellite tracking requirements.

\section{Asteroid Science Requirements}

A notional set of requirements has been established for NESS, based on the asteroid science objectives that are being examined in the current mission concept study. These have assumed that NESS will employ a satellite whose design is similar to that of MOST, which will be equipped with detectors similar to those used in ground-based asteroid tracking observatories, and whose operations will be based on the techniques used currently in ground-based asteroid tracking.

- The NESS science payload will consist of a telescope, 3 CCD detectors (one for tracking science observations, the second for colorimetry science observations, and the third for attitude control star-tracker measurements) and a data system.

- In order to achieve sufficient astrometric precision, the telescope must have a point spread function of FWHM $\sim 1$ arcsec.

- In order to be sufficiently productive in performing follow-up tracking, it must have throughput sufficient to detect asteroids of $\mathrm{m}_{\mathrm{R}}=18-19$ with $\mathrm{S} / \mathrm{N}=2$ in a 600 second (combined) exposure in tracking mode.

- The requirements for colorimetry include the ability to record $\mathrm{m}_{\mathrm{R}}=18$ targets with $\mathrm{S} / \mathrm{N}=20$ in a 1 hour (combined) exposure.

- The telescope must have an unvignetted field of about 1 degree.

- The optical surfaces must be sufficiently smooth that they do not produce unacceptable scattering and the instrument must be baffled in such a way as to allow 
observation within 20 to 45 degrees of the Sun.

- The detectors must be compatible with the above. Astrometric reduction requires that the image scale be 3.5 arcsec/pixel or better. The tracking detector must be approximately $1000 \times 1000$ pixels to cover the required area. It must be capable of exposure times ranging from 1 second to 3600 seconds (combined).

- Spectral bandpasses: the tracking detector need only be sensitive in the visual to red regions $(500-750 \mathrm{~nm})$. The colorimetry detector must have sensitivity in the spectral range $400-1100 \mathrm{~nm}$ in order to cover the range of the ECAS filter scheme.

- The data system must be able to acquire targets in accordance with an uploaded file of target search coordinates or pre-planned search area.

- Positioning of the system requires a tolerance of approximately 20 arcsec. It also requires the ability to record the time of the beginning of each integration to within 1 second of time.

- It must be able to store a minimum of 20 MBytes of data on board and transmit it to a ground station on command

\section{Satellite Tracking Requirements}

The notional requirements for the satellite tracking element of NESS's mission are driven by a basic requirement to be able to detect the majority of the Deep Space Objects in Space Command's catalog of Resident Space Objects, while achieving a astrometric accuracy compatible with catalog-maintenance requirements.

Photometric Sensitivity: Statistics can be gleaned from the Space Command satellite catalog on Deep Space Object sizes and magnitudes. Assuming that NESS, like MOST, will be in a low Earth orbit, these statistics can be analysed to determine the brightness of these objects as seen from this orbit. The bulk of these targets will have a brightness as seen by NESS ranging from $M=11$ to $M=14$.

Target Proper Motion: Both NESS and the target objects will be moving in their own orbits around the Earth, creating a relative velocity between them. This will result in an apparent motion of the target across the sky, as seen by NESS's instrument, of up to 50 arc-seconds per second for a target in geostationary orbit. This will limit the amount of time that the target's image will dwell on any one pixel in the instrument's camera, setting a limit on the amount of signal achievable at the pixel level.

Astrometric Accuracy: NESS should be able to collect images of satellites with a resolution high enough to be compatible with the accuracy needed for the Space Command catalogmaintenance activity, about $1 \mathrm{~km}$. At the $\sim 40,000 \mathrm{~km}$ distance between LEO and GEO, this translates to an image resolution of better than 5 arc-seconds.

\section{NESS System Description}

The NESS mission requirements appear to be achievable using a microsatellite whose design is closely based on the design for the MOST satellite: the same bus with some new software, and the same instrument with some small changes to the layout of the focal-plane of the camera. That being said, there are some improvements in performance for this mission that could be achieved via additional design changes. These are currently being studied by the NESS team, to see which (if any) changes have sufficiently large incremental benefits in terms of performance, to justify the costs of additional re-engineering of the existing MOST design.

For this reason, the best way to describe NESS is to begin by summarizing the MOST satellite design, which is done below; further details 
(although somewhat out of date) can be found in the 1998 MOST paper ${ }^{1}$, and at http://www.dynacon.ca/most.html. This is followed by a summary of NESS issues that are being studied, with discussion of the potential design changes that they could motivate.

\section{MOST System Description}

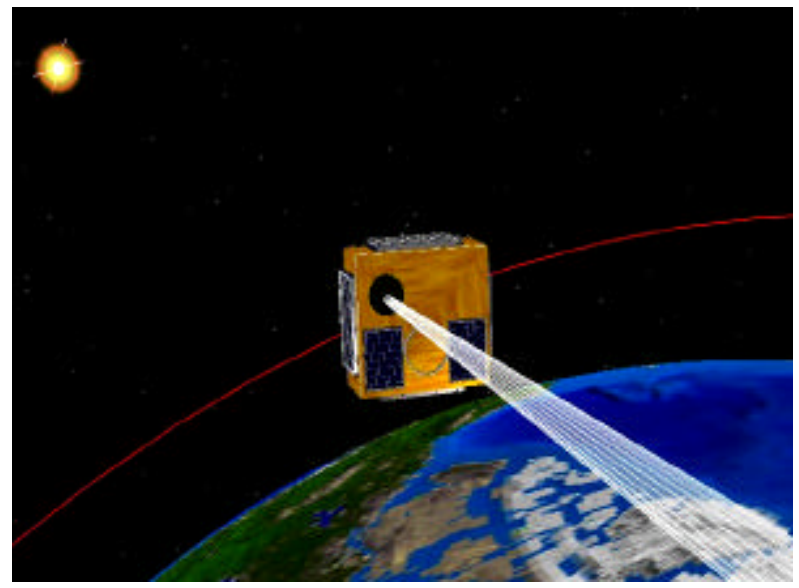

Figure 1: The MOST Microsatellite

\section{Mission Overview}

The science mission for MOST is to conduct very precise, long-duration (several weeks per data record) photometric measurements of stars. The science team, led by P.I. Jaymie Matthews of UBC, will analyze those data to detect stellar oscillations, from which a primary science outcome could be an independent determination of the core composition and ages of stars, and thus a lower limit on the age of the Universe. (There are also numerous secondary science objectives, which are described at http://www.astro.ubc.ca/MOST/index.html.)

Primary funding for MOST is provided by the Space Science Branch of the Canadian Space Agency. Dynacon is prime contractor for the mission, and is providing the ACS and power subsystems. UBC and CRESTech's ISL are providing the science instrument. The satellite is being integrated at the Space Flight Laboratory at the University of Toronto, which is also developing the bus structure, thermal, $\mathrm{OBC}$ and
T\&C subsystems and the ground stations. The Critical Design Review for MOST was completed in the spring of 2000, and the satellite is now being built.

As illustrated in Figure 1, this mission is being implemented using a visible-light telescope instrument on a microsatellite platform, which will be controlled via a pair of ground stations in Canada. The bus size is about 60 by 60 by 20 $\mathrm{cm}$, and the satellite mass is $57 \mathrm{~kg}$.

MOST will be launched as a secondary payload. A launch opportunity in early 2003 has been confirmed (on a Boeing Delta II launcher, with Radarsat 2), with an early-2002 launch option also being studied. To accomplish the science objectives, MOST needs to be in a "dawn/dusk" sun-synchronous orbit (whose plane crosses the equator at $6 \mathrm{Am}$ and $6 \mathrm{PM}$ local time).

\section{Instrument}

The heart of MOST is an astronomical science instrument, shown in Figure 2, that is capable both of astrometrically precise imaging, and of ultra-precise photometry. It consists of:

- A 15-cm aperture, f5.88 Maksutov-optics

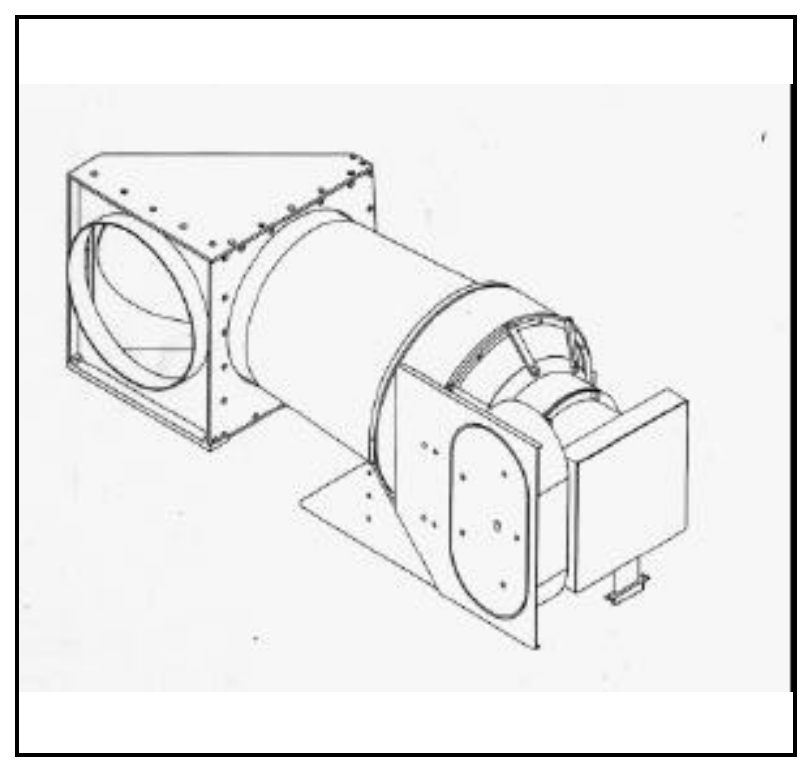

Figure 2: The MOST Instrument 
telescope with an unvignetted field of view of about 0.7 degrees, and a vignetted FOV of about 2 degrees.

- A periscope mirror, to orient the instrument's line of sight correctly with respect to the satellite's frame.

- A camera that includes a pair of identical, $1024 \times 1024$ pixel $(0.86 " \times 0.86 ")$ frametransfer CCD detectors, one of which will be used for photometry science, and the other for collection navigational star-tracking images. It also includes the amplifiers for the CCDs.

- For each CCD, a DSP-based $\boldsymbol{C C D}$ controller electronics board. One of these boards is used to operate one of the CCDs to carry out photometry observations; the other one implements ACS star tracker software

- A set of Fabry lenslets covering a portion of the photometry $\mathrm{CCD}$, which reduce photometric sensitivity to line-of-sight errors. The focal plane arrangement is shown in Figure 3; the inner and outer circles are the unvignetted and vignetted fields of view.

- A focal-plane temperature control system, which includes a passive cryo-cooler to produce focal plane temperatures of $\sim-40 \mathrm{C}$, along with an active temperature control

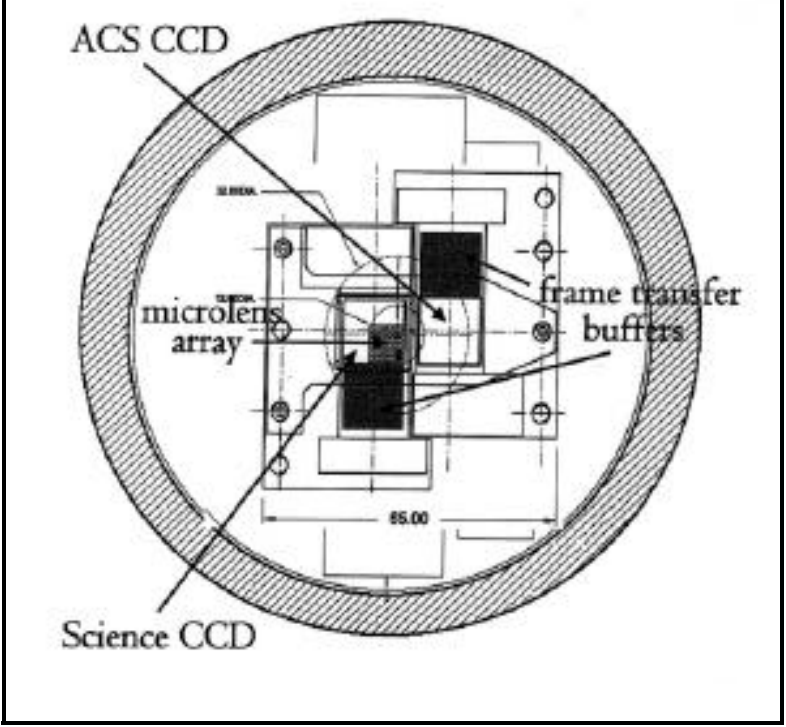

Figure 3: MOST Camera Focal Plane Layout system to regulate detector temperatures to much better than $1 \mathrm{C}$.

- An aperture door, which protects the instrument's optics from contamination by dust during launch, and which can be actuated closed to protect the focal plane from heating by the Sun during detumbling and safe-hold modes.

The mass of the instrument is $15.8 \mathrm{~kg}$, and its power consumption is $8 \mathrm{~W}$.

\section{Bus}

Structure and Thermal Subsystems: The MOST bus structure, shown in Figure 4 in exploded view, comprises a stack of milledaluminum trays, each containing either electronics boards or attitude control sensors and actuators. The instrument is fastened to one side of this stack. This assembly is then covered over by a set of 6 panels, onto which solar arrays, patch antennas, magnetometers and other external equipment are mounted. A marmanclamp payload attach fitting, also fastened to the tray-stack, provides the interface to the launch vehicle. The thermal control is primarily passive, involving selective use of surface finishes and MLI, but it does include battery heaters for use in safe-hold mode.

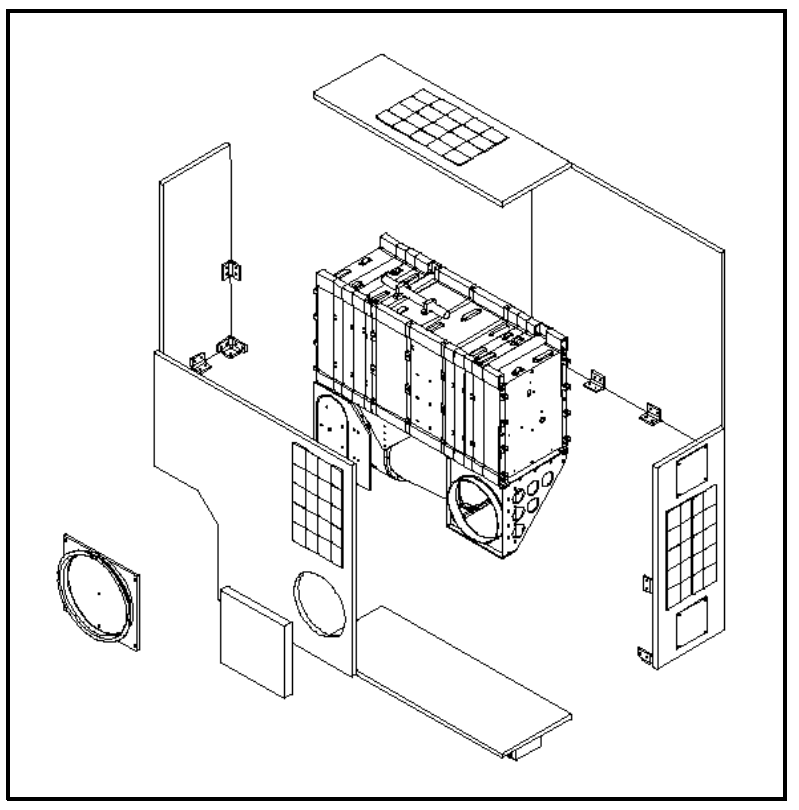

Figure 4: Exploded View of MOST 
Attitude Control Subsystem: The ACS for MOST has been designed to the challenging requirement of keeping the instrument pointed towards specified target stars, with an accuracy of better than 25 arc-seconds, for up to 7 weeks at a time. The design and performance of the ACS is described in detail in a companion paper $^{3}$. In summary, it comprises:

- 3 Dynacon MicroWheel reaction wheels.

- 3 solid-state angular rate sensors.

- 2 dual-wound rod magnetorquers.

- 2 sun sensors.

- 2 Dynacon MicroNode DSP-based attitude control computer boards.

- Dynacon-developed star-tracker software, running on one of the instrument's CCD electronics boards.

- ACS software, running on the MicroNodes, based on modules from Dynacon's MicroDirector ACS software library.

The ACS includes software to detumble the satellite after release from the launch vehicle, to carry out coarse (about 2 degrees accuracy) pointing prior to enabling the star tracker function, to slew from one target star to the next, and to carry out fine pointing when conducting science observations.

Power Subsystem: MOST collects power via solar panels mounted on all 6 faces of the satellite. These use high-efficiency silicon photovoltaic cells. Peak-power tracking is done independently for each panel. Power is stored in a NiCd battery, at a bus voltage of $12-14 \mathrm{~V}$. Switched bus voltage is distributed to each satellite tray; power conditioning is done in a distributed fashion in each tray.

MOST's dawn/dusk sun-synchronous orbit has the desirable property that, for most of the year, the Sun is not eclipsed by the Earth; even during the height of 10-week eclipse season, the maximum eclipse duration is $17 \%$ of the orbit period. Outside of eclipse season the power subsystem produces an average of at least $30 \mathrm{~W}$ when doing science pointing. (Paradoxically, because the solar arrays run cooler during eclipse season, this number increases despite the reduced amount of available sunlight, to $33 \mathrm{~W}$.)

On-Board Computer Subsystem: MOST employs a distributed-computing architecture, with digital processors embedded in almost all the subsystems. The OBC provides communications between all of these, coordinated by a central NEC-V53 processor board equipped with a 16 Mbyte RAM-disk. The RAM-disk is used to store science and telemetry data in the intervals between ground station passes, as well as to store the V53's operating software. The V53 communicates with the ACS MicroNodes and the CCD drive electronics boards via several RS-422 and RS485 serial buses. It also operates the T\&C Subsystem's radios.

Telemetry and Telecommand Subsystem: MOST is equipped with $2 \mathrm{~S}$-band receivers and 2 S-band transmitters, each pair covering one hemisphere of the satellite's field of view, and each using its own patch antennas. The receivers are equipped with 9600 baud modems, and the transmitters with modems that can select between 9600 and 38400 baud.

\section{Ground Stations}

MOST will communicate with two ground stations in Canada, one in Toronto and one in Vancouver. MOST's orbit is such that it will pass within sight of any point on the ground 2 or 3 times near 6 A.M. every day, and 2 or 3 times near 6 P.M. every day, with an average pass lasting 5 to 10 minutes; the geographic separation between ground stations will increase the total daily contact time somewhat.

The two ground stations will be identical. Each employs a 2m-diameter gimballed tracking antenna, driving doppler-corrected S-band transmitters and receivers. These are connected 
through modems to PC-type computers, which carry out tracking, telemetry and command functions, and which collect and forward to the operations team the science data and engineering telemetry data collected each day.

\section{Performance of the "MOST-As-Is" Design}

Initial analysis of the MOST satellite designindicates that, with minor modifications, it would be able to carry out asteroid and satellite tracking operations, at a performance level that approaches the requirements of the NESS mission. These changes are:

- Removal of the Fabry lenslet array in the focal plane of the camera. These lenslets are needed to carry out MOST's photometry mission, but for NESS they merely obscure otherwise-useful CCD area.

- Modify the software in order to carry out some simple image-processing, in order to either send raw camera images to the ground for processing, or to carry out some level of asteroid and satellite tracking processing aboard the satellite.
This would result in a satellite that could take useful satellite tracking images and asteroid tracking images. It would not enable asteroid compositional studies; additional of colour filters and polarizing filters would be needed for that.

The graph in Figure 5 can be used to determine the level of asteroid and satellite tracking performance achievable by the MOST design "as is." It presents the results of an a photometric analysis of the MOST instrument, when operated in a mode compatible with asteroid and satellite tracking operations:

- The satellite is assumed to be pointing in an inertially fixed direction, held by the ACS. The effects of pointing error/jitter are neglected in this analysis.

- A target asteroid or satellite is assumed to be drifting across the field of view of the instrument. The rate of drift, in arc-seconds per second, represents one axis of the graph.

- The faster the target's proper motion, the less time it will spend depositing light energy into any one pixel of the MOST camera's CCD, resulting in a lower signal

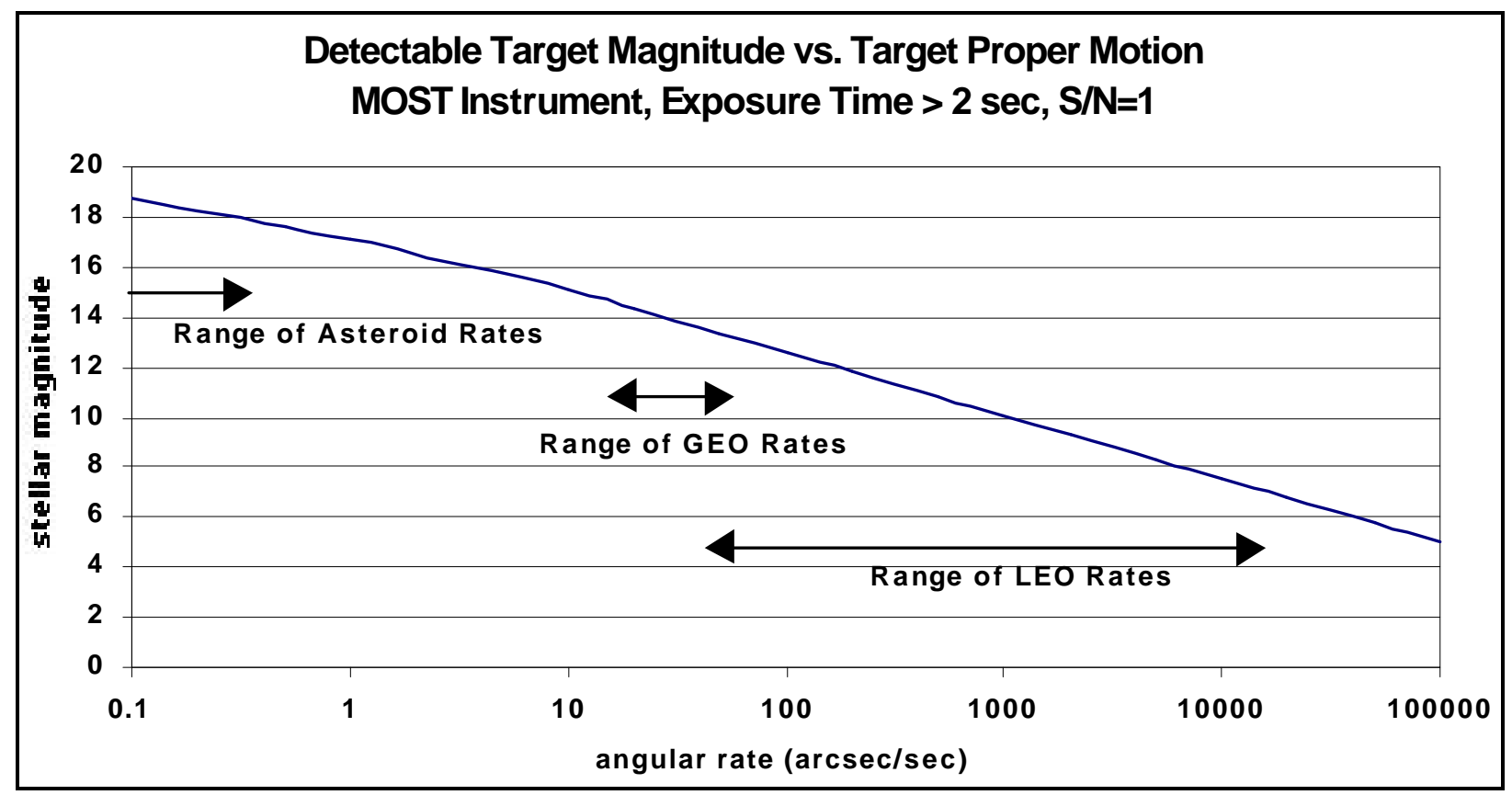

Figure 5: Photometric Performance of MOST Instrument in Tracking Moving Targets 
level.

- The graph shows the brightness that a target must have, expressed in terms of equivalent stellar magnitude, in order to result in a signal to noise ratio of 1 at the pixel level, taking into account the known sources of noise in the MOST instrument.

- An exposure time of 2 seconds was chosen, relatively arbitrarily, for most of the cases analyzed. For proper motion values below 1.5 arc-seconds per second, the exposure length was increased to take advantage of the longer dwell-time of the target in each pixel. The curve in the graph represents an exposure-length optimization in which readout noise is traded against dark-current noise (among many other effects).

The result is that the slower the target's proper motion, the fainter the target can be in order to produce a given S/N. MOST's instrument is capable of achieving an $\mathrm{S} / \mathrm{N}$ of 1 for target satellites in GEO as faint as $\mathrm{M}=13$ to 15 . This $\mathrm{S} / \mathrm{N}$ level can be achieved for asteroids as faint as $\mathrm{M}=18$ to 19 .

These numbers do not translate directly in to target detectability levels. To detect a target, an image consisting of a large array of pixels must be analysed, and compared with other images to detect a moving target. The algorithms for doing this are quite complicated; while they are critically affected by the $\mathrm{S} / \mathrm{N}$ achieved at the pixel level, they are also subject to other factors. An S/N of 1 reflects a lower limit, below which targets could likely not be detected using pairs of images. However, some noise suppression can be achieved by combining images taken in rapid succession. Thus, an $\mathrm{S} / \mathrm{N}$ of 1 at the pixel level could, with the right post-processing, be a fair proxy for the level at which a target is detectable.

With those caveats, how does the photometric capability of MOST's instrument compare with the two main NESS requirements?
- The DND's SOS mission is expected to be used to track targets in GEO and higher orbits. The great majority of these have a visual magnitude of $M=11$ to 14 . From the graph, it appears that MOST's instrument is suitable for detecting these, with suitable image processing. Thus, the MOST design should be suitable for carrying out SOSrelated research in the NESS mission.

- The NESS asteroid-tracking requirement is to be able to detect asteroids of magnitude $\mathrm{M}=18$ to 19 , with $\mathrm{S} / \mathrm{N}=2$ in a 600 second (combined) exposure. The MOST instrument comes within about 1 magnitude of being able to achieve that, using single exposures with a typical exposure length of 2 to 40 seconds. The analysis has not yet been done to factor in the effect of combined exposures; this is expected to improve performance.

\section{NESS Design Issues}

The above analysis is not intended to argue that the NESS mission should be flown using the MOST design without modifications. While making any modifications to the design would incur some non-recurring engineering costs, it is almost certainly the case that there are modifications that would significantly improve performance, thus increasing the value of the mission, both from the asteroid-science and the satellite-tracking R\&D perspectives. Analysis to quantify these costs and benefits is currently being done by the NESS team.

This analysis is focusing on several issues with the MOST design, which affect the ability of that design to support some of the NESS mission requirements, or affect the performance achievable using that design. These are discussed in this section, along with design trade-offs that are being investigated. 


\section{Camera Photometric Sensitivity}

The photometric sensitivity of the MOST instrument appears to be adequate for the satellite-tracking $\mathrm{R} \& \mathrm{D}$ mission. However, it is on the low side when compared to the asteroid tracking requirements. If sufficient sensitivity failed to be achieved, then the minimum size of asteroids that could be detected would be fairly large, resulting in a sharp drop-off in asteroid tracking opportunities.

One remedy for this would be to increase the aperture size of the instrument; as discussed under "Field of View," below, this could be both achievable and desirable. Other approaches being investigated include on-chip binning of pixel signals, and on-board signal processing to allow noise-suppression via temporal filtering using multiple short exposures.

\section{Instrument Field Of View}

The field of view of the MOST instrument is a bit narrow for both asteroid-tracking and satellite-tracking applications:

- For satellites, there is some uncertainty in the catalog location of the object, and a narrow FOV results in a large probability that the FOV of the instrument will miss the satellite when the instrument is targeted towards it. Also, the high proper motion rates for satellites means that a narrow-FOV instrument has difficulty encompassing a complete satellite track image with an exposure of reasonable length.

- For asteroids, a larger FOV would increase productivity of NESS in asteroid-search mode.

The detectors in the MOST instrument cover $0.86 " \mathrm{x} 0.86$ " of the sky. Increasing this to about $2 " \mathrm{x} 2$ " could satisfy both mission requirements. This could be done by altering the lens and secondary mirror of the telescope to decrease its

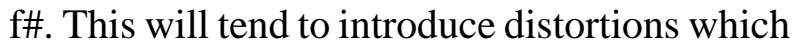

will interfere with the ACS star tracking function. However, if the aperture is increased at the same time, the $\mathrm{f} \#$ could be decreased while reducing distortions.

This could be accommodated with the current MOST volume envelope. The MOST telescope aperture diameter is smaller than the available space, by several $\mathrm{cm}$, in order to simultaneously achieve a narrow FOV (which reduces photometric noise and improves star tracker performance and hence ACS accuracy) and low distortion. If a larger FOV is sought, that extra volume could be used to allow an aperture increase. (This applies if NESS ends up flying on a Delta II launcher, like MOST; on another launcher, a new volume accommodation for the satellite would alter this trade.)

Increasing the aperture has the desirable effect of allowing more photons to be collected per second, improving S/N. Negative effects include increased instrument mass, and a reduced astrometric accuracy, which will reduce both the resolution of the star-tracker (and hence the attitude controller), and of the resulting asteroid and satellite tracking data.

\section{Satellite Layout and Baffling}

In order for NESS to be able to track Aten asteroids it must be able to operate while pointing fairly close to the Sun (from 45 to 90 degrees from the Sun). MOST as currently laid out is designed to have the instrument point almost directly away from the Sun, while its main solar array is pointing almost directly at the Sun.

To target NESS towards Aten asteroids while collecting enough solar power will require the satellite's layout to be changed. An obvious change is to eliminate the instrument's periscope mirror, and move the aperture to the side adjacent to the main solar array; then when the instrument is pointing between 45 and 90 degrees from the Sun, the solar array will point to within 45 degrees of the Sun, an almost 
optimal power-collection scenario. This has the added benefit of eliminating the periscope mirror's considerable mass. In this approach, the satellite's body can also continue to act as a baffle; additional baffling is expected to also be necessary, to reduce photometric noise from stray sunlight.

\section{Filters for Colorimetry and Polarimetry}

The MOST instrument is equipped with a single fixed filter, and so cannot be used to carry out any asteroid compositional studies. A straightforward approach to correcting this is to add a filter wheel, which could switch the 8 standard ECAS filters, as well as polarimetric filters, into the detector's field of view. However, this is likely to be a relatively expensive option; also, placing significant equipment into the telescope optics path will increase stray light, and hence photometric noise, reducing achievable $\mathrm{S} / \mathrm{N}$ values.

Another alternative will also be examined: placing strips of filter and/or polarizing material directly over small portions of one of the detectors. This could involve adding a third detector to the camera's focal plane, which would likely mean re-arranging the other two detectors. The third detector could be identical to the first two; or, it might be mor desirable to use a detector with a wider spectral response bandwidth, to more effectively support the IR bands of the ECAS colorimetry measurements. An additional detector plus filter might add $1 \mathrm{~kg}$ of mass, and $2 \mathrm{~W}$ of power consumption to the instrument.

\section{On-Board Image Processing}

Satellite and asteroid tracking, as done from ground-based observatories, both use similar approaches to detect moving objects against the sky background. Multiple images are collected, then mathematically registered against each other. Some of them may be combined together to increase signal levels. They are then compared, to look for star-like objects that move between two images taken at different times. Or, single images or sets of images that were taken with longer exposures are compared to look for streaks from moving objects (which must be discriminated from streaks from noise sources).

A straight-forward way to operate NESS, then, would be to have it collect images taken in commanded directions for commanded exposure lengths, and download these to the ground for processing using existing processing facilities. This is undesirable, however, because each image consists of a large amount of data, enough to quickly fill the data downlink capacity of the MOST-design radios.

One way to overcome this limitation would be to modify the radio design to increase downlink capacity. Some of this may be possible via higher-gain antennas on the satellite and on the ground, and via a higher-power transmitter on the satellite...which in turn could be accommodated by either larger solar panels, or via switching from silicon to GaAs solar cells.

Some relief could come from image compression before downlink. However, while star-field images appear sparse enough to be excellent candidates for compression, in order to avoid filtering out faint targets some of the noise in the image will end up being included, reducing compression efficiency. Investigations are being carried out to quantify compression ratios achievable in these circumstances.

An alternate approach is to reduce the amount of data that needs to be downlinked to the ground, by moving some of the image processing tasks into the satellite's data processing system. The MOST design includes very powerful (80 MIPS) DSP-based processor boards with large (32 Mbyte) RAM-disks, that should be able to carry out extensive image processing and storage. An additional processor can be carried to increase this capacity further, at a small power $(\sim 1.5 \mathrm{~W})$ and mass $(\sim 0.5 \mathrm{~kg})$ penalty. The 
algorithms currently being used in ground-based processing are now being studied to determine what subset of them could be carried out autonomously on-board NESS, and the effects this would have on downlink data volume.

\section{Orbit}

The MOST requires being launched into a dawn/dusk sun-synchronous orbit, in order to be able to achieve science requirements. Since few primary payloads fly into this orbit, this has reduced MOST's launch options as a secondary payload. A desirable side-effect is that MOST's power subsystem does not have to accommodate long eclipses, allowing smaller batteries and solar arrays.

It currently appears that the NESS mission could be carried out from a much wider range of orbits. For logistical and cost reasons, then, NESS may fly in a different orbit than MOST. However, any other orbit will result in relatively long eclipses, requiring a larger battery and higher-power solar arrays than MOST. The latter could be accomplished either via increasing the size of the solar panels, or by switching from silicon to GaAs solar cells (the latter having a significant cost impact).

\section{Attitude Control Performance}

MOST is relatively insensitive to jitter, as long as attitude errors stay within a 25 arc-sec bound. NESS will want either more-accurate attitude control for long exposures, or else will accept the current control accuracy but will then need to carry out shorter exposures, which then would either have to be compressed and downlinked directly (increasing the magnitude of the data downlink capacity issue), or would have to be processed on-board to register and merge these.

\section{Development Plan}

The development team for the NESS satellite is expected to be the same as that for MOST, consistent with the very close similarity between NESS and MOST; indeed, one attractive costreducing option is to coordinate the construction of MOST and NESS, so that elements of both satellites are built at the same time, with both programs possibly sharing spares, etc.

A separate science team has been formed for the NESS mission. The team is led by Principal Investigator Alan Hildebrand, who is also leading asteroid compositional study investigations. David Balam is leading asteroid tracking investigations. Jaymie Matthews will lead instrument development, and is lading instrument-related design and trade-off studies. Dynacon is currently carrying out satellitetracking R\&D related work; participation from others in DND is also expected. During the course of the present concept study, other asteroid researchers in Canada and abroad are expected to join the science team.

The Space Science Branch of the Canadian Space Agency is supporting the science team, providing funding via their Concept Studies Program.

Dynacon has been contracted by the Defence Research Establishment of the Canadian Department of National Defence (DND), to study how a microsatellite based on the MOST design could be used to conduct experimental satellite-tracking activities.

It is currently expected that NESS could be developed within a schedule as short as 2 years, depending on how closely it can be coordinated with the MOST development schedule.

The development cost of NESS will depend on two main factors: the extent to which it is decided to modify the MOST design to increase NESS performance and functionality, and the 
extent to which NESS's development can be coordinated with the development of MOST. If coordination can be done near-optimally, and a minimal re-design option is selected, the cost could be somewhat less than that of MOST (MOST will cost the Canadian and Ontario governments approximately CDN\$7M, or about US $\$ 4.5 \mathrm{M})$. More-extensive re-design, or a development that is not coordinated with that of MOST, would likely cost abou the same as MOST.

\section{Acknowledgements}

The Space Science Branch of the Canadian Space Agency is funding the NESS science team to study the asteroid-science aspects of the mission, under the supervision of Roger Colley. The Defence Research and Development Branch of the Canadian Department of National Defence has provided support to Dynacon for analysing the potential for using a microsatellite-based system to collect and process images for satellite-tracking purposes. This work has been supervised by Lt.-Col. (Ret.) Philip Somers, and by Lt. Claudia Vachon.

\section{$\underline{\text { References }}$}

1. Carroll, K.A., Zee, R.E., Matthews, J., "The MOST Microsatellite Mission: Canada's First Space Telescope," Proc. $12^{\text {th }}$ AIAA/USU Small Satellites Conference, Paper No. SSC98-VI-6, Logan, Utah, Aug. 31-Sept. 3 1998.

2.Carroll, K.A., McTavish, D.J., Matthews, J. and Rucinski, S., "MOST: A Space Astronomy Microsatellite Mission," presented in poster session at the $11^{\text {th }}$ AIAA/USU Small Satellites Conference, Logan, Utah, Sept.15-18, 1997.

3. Grocott, S.C.O., "Modular Attitude Control System for Microsatellites with Stringent Pointing Requirements," Proc. $13^{\text {th }}$
AIAA/USU Small Satellites Conference, Paper No. SSC00-VIII-6, Logan, Utah, Aug. 21-24, 2000.

4.Stokes, G.H., "SBV Program Overview," Proc. 1997 Space Control Conference, 25-27 March 1997, MIT Lincoln Laboratory. 